\title{
Challenging care experiences among caregivers of hemodialysis patients
}

\author{
Fatemeh Ghapanvari ${ }^{1}$, Seyedehzahra Hosseinigolafshani ${ }^{2, *}$ \\ ${ }^{1}$ Student Research Committee, Qazvin University of Medical Sciences, Qazvin, Iran \\ ${ }^{2}$ Social Determinants of Health Research Center, Qazvin University of Medical Sciences, Qazvin, \\ Iran
}
*. Corresponding author: Seyedehzahra Hosseinigolafshani, Social Determinants of Health Research Center, Qazvin University of Medical Sciences, Qazvin, Iran. E-mail: z.hoseinigolafshani@qums.ac.ir.

Cite this article: Ghapanvari, F.; Hosseini Golafshani, S.Z. Challenging care experiences among caregivers of hemodialysis patients. Int J Epidemiol Health Sci 2021;2(9):e19. Doi:10.51757/IJEHS.2.9.2021.245203.

\begin{abstract}
Background: Recent studies on hemodialysis patients have focused more on the suffering caused by physical and psychological problems in them, and they have paid less attention to their caregivers. Since the caregivers of these patients are intensively affected by the physical and psychological problems caused by this method of treatment, it is necessary to pay more attention to the challenges as well as the problems that exist among them. This study aimed to describe the lived experiences of caregivers in caring for hemodialysis patients.

Methods: The present study is a qualitative study with a phenomenological design. Sampling was first done through a purposive sampling method, and the snowball sampling method was then applied in order to select 21 caregivers for the hemodialysis patients from three hemodialysis centers in Qazvin, Iran. An audio recorder were used to collect the data of in-depth face-to-face semi-structured interviews. The interviews were analyzed using Colaizzi's approach. The criteria of Lincoln and Guba was followed for trustworthiness.

Results: Analysis of the interviews in response to the main question revealed 108 initial codes, 18 subcategories, and 3 main themes, including 1. Care: Growth experience, 2. Care: Living in solitary confinement, and 3. In silence: Seeking help.

Conclusion: In situations where the caregivers of hemodialysis patients felt they did not have sufficient material and spiritual support from those around them, they reported two different and conflicted experiences. Some people experienced progress and excellence, while others felt stuck and had to make ideal sacrifices. More in-depth research is required to discover the factors that influence such diverse insights.
\end{abstract}

Keywords: Challenge; Care experience; Caregiver; Hemodialysis; Patients; Patient care

\section{Introduction}

To manage chronic kidney disease, several methods of treatment, such as hemodialysis, peritoneal dialysis, and kidney transplantation, are available (1, 2). Among them, hemodialysis, for various reasons, is the most common method of choice worldwide $(1,3,4)$. According to global statistics, by the end of 2016, the number of patients undergoing dialysis was estimated to be $2,989,000,89 \%$ of which were hemodialysis patients (3).

Although the widespread availability of hemodialysis saves and prolongs the lives of thousands of patients with chronic kidney disease, this complex method of treatment can in fact result in fundamental changes in various dimensions of a patient's life (6). Long-term hemodialysis therapy often leads to high dependence 
of patients on caregivers, families, and healthcare systems (7).

Caring for patients with chronic diseases is usually done by their family members, mostly spouses, parents, and siblings (8). The involvement of the caregivers in patients' transfer to the hemodialysis ward, the preparation of appropriate foods, meeting the health needs, paying attention to and managing the side effects of the hemodialysis, monitoring patients' vital signs (7), and providing emotional and psychological support (9) impose a massive burden on the caregivers (7). The results of previous studies revealed that caregivers of patients with chronic diseases suffer from various physical and mental problems, and show various psychological symptoms such as depression, anxiety, anger, despair, and feelings of guilt and shame (5).

In addition, enduring such an additional burden leads to several changes in their daily relationships, social interactions, and work (10). Under these circumstances, most caregivers give superiority to the patient's needs compared to their own, and, as a result, spend less time taking care of their own health-related matters. This hasty prioritization, in turn, will have adverse effects on their own health and well-being (6).

The prolonged physical and psychological strain has led to certain issues with this group of people being viewed as a serious health issue in some areas, with caregivers being referred to as "hidden patients" in some cases $(5,9)$. The professional approach to comprehensive support of these people is to understand their insights, interactions, and experiences in caring for their patients at home as well as in the hemodialysis ward (11). Understanding their experiences can be a significant contribution to family-centered interventions, including increasing the preparation of patients and their caregivers for alternative treatments (11).

Most studies in this regard were conducted using a quantitative and a positivism approach, and they used predetermined instruments. This is while the depth of the problems, the challenges, the quality of the hidden interactions, and the physical as well as the psychological burden on the caregivers will be understood only through a qualitative approach, open interviews, devoting more time, and immersion in their daily experiences. Therefore, the current study was designed and conducted with a qualitative phenomenology approach to deeply understand the care experience among the caregivers of hemodialysis patients. The aim of this study was to describe the challenging care experiences of caregivers in caring for hemodialysis patients.

\section{Materials and methods}

\section{Design and Setting of the Study}

The present study is a qualitative study with a phenomenological design. Phenomenology is a qualitative method which tries to determine the fundamental features and structures of life experiences in a regular and reciprocal way, and the researcher's goal is to understand the meaning of these experiences in the same way that the participant has experienced them (12). The study setting were three hemodialysis centers located in this city which provide hemodialysis services to patients.

\section{Description of Participants}

In qualitative research, sampling is done through a purposive sampling method in which the researcher relies on his/her judgment when choosing the samples (13). Accordingly, in the current study, 21 caregivers, who all met the inclusion criteria, were selected in the first stage through a purposive sampling method, and then, through a snowball sampling method. The inclusion criteria were: having consent to participate in the study, being responsible for direct care of the patient for at least six months, aged at least 18 years old, not receiving any payment for the care, not having a history of any known mental illness, and not taking any psychotropic drugs (according to the samples' statements), not being responsible for the care of another patient, being able to read and write, and being able to communicate verbally.

\section{Data Collection}

In the current study, the data was collected through semi-structured interviews. The interviews began with the least structure, and they gradually became more structured. The interviews were digitally recorded then transcribed verbatim. All interviews were conducted by the main investigator. If necessary, the researcher also used the participants' memories, poems, and writings about their lives, as well as the filed notes. During the interviews, if necessary, probing questions were asked, based on the participants' previous responses, in order to encourage them to describe their 
feelings in greater detail (14). With the progress of the study and the emergence of the descriptions, the data collection continued until the data saturation was reached when no new description of the phenomenon was provided (13-15). To encourage the participants to share their experiences, the researcher used the following questions:

- Please tell me about your experiences as the caregiver of a hemodialysis patient.

- How does caring for your patients make you feel?

Each interview lasted between 45 and 60 minutes, depending on the participants' tolerance. The interviews were conducted in a meeting room, located in the study setting, which was a quiet place away from any noises. The interview was considered completed if the participants described their experiences and there was no need to clarify the matter. If, after reading the first interview, an additional interview was needed to clarify the matter, a second interview would be conducted.

\section{Data Analysis}

For data analysis, the Colaizzi's method was used by performing the following steps:

During the first step of this method, at the end of each interview and taking the field notes, the recorded statements were first repeatedly listened to, and their statements were then transcribed verbatim on a piece of paper and read several times to understand the participants' feelings and experiences. In the second step, after reading the participants' descriptions, and feeling and understanding their emotions, the meaningful information as well as the statements related to the phenomenon discussed were underlined. By this means, the significant statements were identified. During the third step, which is to extract the formulated concepts, an attempt was made to extract a concept from each statement after identifying the significant statements in each interview. These concepts should provide the meaning and the fundamental part of the individual's thinking. In fact, after extracting these concepts, an attempt was made to examine the relevance of these formulated meanings to the main and initial statements, and to ensure the correctness of the relationship between them. In the fourth step, after extracting the codes, the researcher carefully read the formulated concepts and clustered them into themes based on their similarities. By this means, the themes were formed from the formulated concepts. During the fifth step, the concepts were incorporated to comprehensively describe the phenomenon and to create a more general category. In the sixth step, a comprehensive description of the phenomenon without any ambiguity was developed. The final (seventh) step was done through validating and referring to each sample, and asking about the findings $(15,16)$.

\section{Trustworthiness of the Study}

For establishing reliability and validity, the Lincoln \& Guba's criteria, including credibility, dependability, confirmability, transferability, and fittingness, were used (17). Credibility was achieved through the researcher's prolonged engagement with the subject, adequate participation, and appropriate interaction with the samples. The dependability was achieved through writing a quick copy, considering the opinions of the colleagues, and re-reading the whole data. The confirmability was achieved through time combination and a variety of sampling in participants. Finally, for transferability, detailed explanations of the fields and findings were provided (12).

\section{Ethical Considerations}

The current study was approved by the ethics committee of Qazvin University of Medical Sciences (Ethics Code: IRQUMS.REC.1398.352). Before the interviews, detailed explanations of the study and its process were provided to the participants. All the participants signed the informed consent form. The participants were assured that the collected data would only be used for academic purposes, and the data, along with their names and details, would be kept confidential. The researcher used some codes to present the data. All the data was stored on the researcher's personal computer.

\section{Results}

Twenty one caregivers of hemodialysis patients (13 females and eight males) took part in the current study. The average age of participants was 49.71 years, and they had been caring for their patients for an average of 3.91 years.

Following analysis of the interviews in answer to the study's core topic, 108 initial codes and 18 subcategories were extracted, which were then 
grouped into three broad themes. The most important themes included: 1. Care: Growth experience, 2. Care: Living in a solitary confinement, and 3 . In silence: Seeking help. Figure 1 shows the themes and the relevant subcategories.

\section{Care: Growth experience}

Although most participants reported feelings of sadness, despair, and hopelessness at the time of a family member's dialysis diagnosis, as well as crisis and shock in some ways, they said that, in the end, facing the various challenges of living with a hemodialysis patient resulted in some degree of growth experience and intellectual maturity in various aspects.

Participant No. 6: "The first time I was told my wife would need dialysis, I was really distraught and cried for several months," says participant No. 6. But then I began to believe that my wife's need for dialysis was God's will. God never shuts one door and doesn't open another. For God's sake, I'm taking care of my wife right now, and I always tell people that we have a good God.

After facing the obstacles and embracing the circumstance, it appears that the participants gave life a new meaning and viewed life and new challenges from a fresh viewpoint.

Participant No. 17: "I am satisfy to be with my mother, in spite of the issues and challenges. Taking care of her doesn't worry me, and also the undeniable fact that she's alive is enough for me".

Participant No. 8: "Everyone in my family thinks I've turned into a legend. I don't want to brag, but this is something that many of my relatives have told me. Because of my patience and the care I received from my father, I became a role model for them. I didn't intend to be a role model, but I unintentionally became one. Maybe it's because of my parents' affection for one other."

The subcategories of this core theme include "the caregiver's spiritual growth," "the development of the caregiver's ability," "successful adaptation," "others' admiration," and "dealing with problems and respecting one's wishes as a component in recharging".

\section{Care: Living in solitary confinement}

Care: The current study's second significant subject was living in solitary confinement. Unlike the previous participants, these participants believed that caring for a hemodialysis patient had resulted in changes in their lives that were associated with feelings of dying, Losing hope, abandoning themselves, and even being forced to reject their inner aspirations and goals, such as marriage, quitting job, and limiting recreation and social activities. And it's as if the caregiver has been entrapped by the caring.

Participant No. 10: " it's difficult... You need to ignore lots of things in these situations... your recreation...your fun...everything... You're sad because you can't celebrate or because once you go somewhere and also the host prepares food and you can't eat it "..

Participant No. 5: "I decided never to marry, to avoid leaving my mother alone. Oh, my mum is going to be really lonely if I arrange to marry. I never want to be alone with her."

Participant No. 7: "Now have a glance at me." I'm also feeling under the weather. This is the state of my leg. My knees have enlarged and are in pain. I'm in so much agony in my legs that they're all bruised from the stress. But I didn't follow up, and I didn't see a doctor. I need to look after my hubby... I don't have time to visit a doctor any longer. "Who wants to look after him? If I go, they'll put me in the hospital for a time." I tell myself.

Participant No. 16: "We come here, every other day." I must make his food, wash the dishes, and tidy the house before I go. After that, I take a nap before getting up to think about what I want to make for supper. As if it were a factory. I'm the housewife and the man of the house. When he arrives for dialysis, he spends four hours here. In order for him not to be alone at home during these four hours, I must go do my personal work, go shopping, and so on. Then we'll all return home together."

"The caregiver's perfect sacrifice," "lasting regret, why us?" are notions that come to mind.

This main topic is divided into elements like "loss of usual routine of life," "continuous caregiver concern," "suffering from viewing the patient's physical condition," "wishing to return to health," and "unsuitable home facilities."

\section{In silence: Seeking help}

While personnel and supervisors at the healthcare system tried to help and give instructional information 
on a regular basis, the training was insufficient, and many of the participants' inquiries about patient care went unanswered. As a result, they were agitated, blaming their patients, and sometimes even themselves, for their current condition, in addition to the healthcare providers. The participants also mentioned that they needed the help and special attention of health officials to deal with the various challenges that hemodialysis patients encounter.

Participant No. 21: "I had no idea what dialysis was at first. What is the need for dialysis in my wife's case? I assumed it was similar to taking a picture, administering a test, or injecting anything. She'd get better soon, and we'd be able to leave... But once I was here, I realized what dialysis was "..

Patients on hemodialysis should be regarded not just as a distinct group of patients, but also as a distinct group of patients, according to the participants.

Participant No. 8: "Dialysis sufferers are sometimes treated as if they were special patients, although they aren't." They're just like any other patient. They specialize in dialysis, which is a free service. In other cases, such as when patients wish to buy their prescriptions, they need to be given the drug confirmation right away and not have to wait in line for a lengthy period. While this isn't true, the patient drives an hour and stands in line for two hours just to acquire his or her medicine approval."

Participant No. 9: "My idea is that the authorities should pay more attention to these people and provide more assistance to them in terms of drugs and other matters." Their medications are prohibitively pricey. We are obligated to purchase their medications. It's not a matter of whether we want to buy it or not; we have no choice. We anticipate assistance from the government. The renal foundation should be able to assist us in dealing with these patients. They pay us a monthly charge for their medicine, for example."

"Inadequate diabetes management," "initial perplexity," "insufficient information regarding care," "the need for other members' aid," and "seeking authorities' support" are all subcategories of this topic.

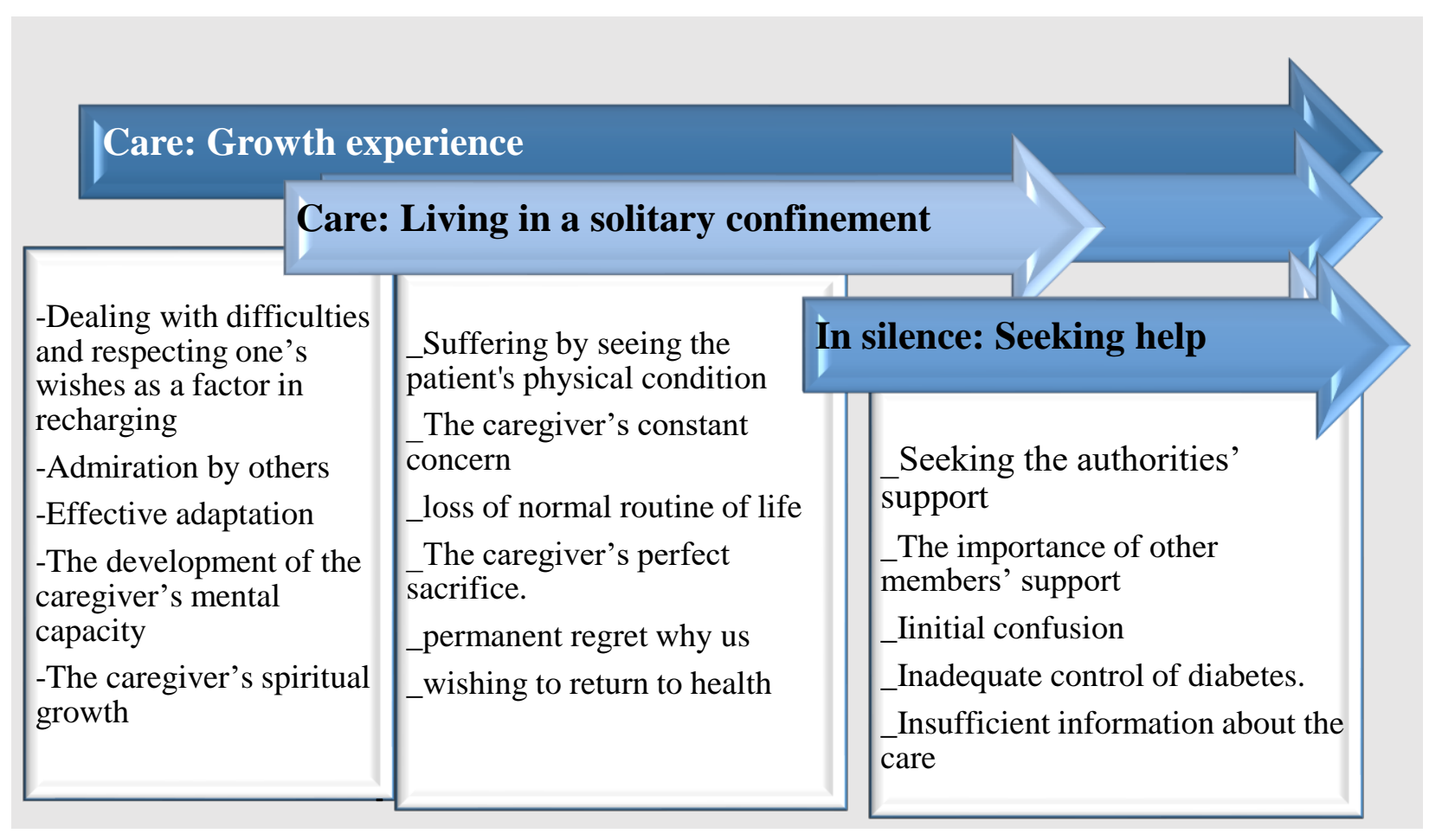

Figure1: Themes and relevant sup categories. 


\section{Discussion}

\section{Care: Growth experience}

Following their initial apprehension about accepting their new role as a caregiver, the participants claimed to have made intellectual growth in terms of spirituality and psychology. The caregivers appear to have come to this conclusion after a long internal struggle and the initial confusion that they felt upon entering a new setting. In this way, they can make the path's continuation easier for themselves. While attempting to reach this goal, some participants were able to achieve some sorts of effective adaptation, while others demonstrated intellectual growth and maturity in other areas. The findings of the investigation by Wightman and colleagues (2019) are consistent with these findings. Personal growth was one of the study's main themes, with a group of pediatric hemodialysis patients' caregivers indicating a wide range of coping techniques for developing and maintaining self-care, including mindfulness, exercise, relaxation, and faith. Others added that their experiences had provided them with wisdom and insight, allowing them to better manage their children's problems in the long run and have a more holistic approach to life and caregiving (18). In a separate study, Hoang and colleagues (2018) discovered that, despite heavy workloads, hemodialysis caregivers have some personal growth, and that this intellectual maturity often aids them in being able to support their patients in difficult situations (19).

According to the interview findings, one of the most effective strategies for most of the participants in the current study to cope with their circumstances was to focus on spiritual issues and give life a new meaning. Having a strong faith and believe in God, as well as gaining his approval, has improved their ability to deal with situations and brought them serenity. These findings are in line with the findings of a study conducted by Khorsandi and colleagues (2020). Appealing to God and heavenly knowledge were subcategories of the major theme of spiritual beliefs in their studies, and they resulted in calm and strengthened their ability to deal with challenges (20).

One of the topics uncovered in Rocha and colleagues' (2018) study was spirituality as a foundation for living. According to the study, caregivers overcome obstacles and challenges and give purpose to their lives by providing meaning to their daily interactions. In this study, loving and caring for the patient were also discovered as significant factors to spirituality (21). In other words, caregivers are better able to deal with the challenges and problems they face because they believe God protects them and that there is a higher power at work in our everyday encounters (22).

\section{Care: Living in a solitary confinement}

The data analysis revealed that the disease's chronic and long-term nature, as well as the patients' need for constant and thorough care, had effectively imprisoned and constrained the caregivers' world to caring for the patients. These findings are consistent with the results of some previous studies $(23,24)$. Family caregivers "no longer live," according to these research, and they lack the ability to use their employment rights to work, get benefits, and associate with their friends and relatives. In such cases, the caregivers bear an added load that comes with a number of major negative re-percussions. The findings of the De Pasquale and colleagues study (2019) also demonstrated that for this group of caregivers, the emergence of unexpected duties leads to negative psychological responses such as anxiety and tension (11).

In line with these findings, Mieto and Bousso (2014) discovered that when a kid is placed on hemodialysis, the mother, as the child's primary caregiver, experiences significant changes in her daily life. Because mothers are responsible for accompanying their children to hemodialysis sessions, the feeling of being trapped with her children within the care of the hemodialysis machine, the need to give up work, the loss of their social place, anxiety about dietary restrictions, fear of their children's impending death, intense isolation, and their inability to communicate

\section{In silence: Seeking help}

Following the disease's diagnosis, the caregivers found themselves alone in a new world, full of ambiguity and bewilderment, according to the analysis of the interviews. They wanted more information from healthcare practitioners about how to care for their patients on the one hand, but they received no significant support or attention on the other. In reality, the current study found that caregivers lacked proper training in a variety of areas of care, including 
nutrition, medicine, weight control, and their patients' symptoms, and that they were frequently nervous and stressed about what to do if any side effects occurred. These findings are in line with Wightman and colleagues (2019) (18) and Tong and colleagues (20) findings. The caregivers of hemodialysis patients in these studies were equally surprised and perplexed at the time of diagnosis, and it was difficult for them to comprehend and accept this truth. One of the primary themes in the De Pasquale and colleagues study (2019) was "treatment delivery and logistics," with subcategories of "insufficient information," "medication regimen," and "logistical issues." As a result, caregiver training appears to be especially important, as caregivers require knowledge, special skills, training, and appropriate guidance from the healthcare team in order to effectively care for their patients. Furthermore, training caregivers on how to care for their patients at home improves their satisfaction as well as their quality of life (27).

In the current study, the participants emphasized the importance of helping and supporting the other members of the family in order to provide higher quality care, and complained about the lack of support from the other family members. These findings are consistent with the results of Pourghaznein and colleagues (2019) study. In their study, one of the themes was "a sense of being unsupported by family and friends". The participants felt that their conditions are not understood by the other family members, and this leads to a feeling of being ignored by them (29). In their study, Mthembu et al. (2016) found that the caregivers did not receive any support and assistance from other family members. It was further indicated that some of the family members are unwilling to share the role of caring for their parents (30).

Another finding of the current study, which revealed that caregivers encounter additional psychological obstacles, is that they anticipate authorities focusing more precisely on the challenges and demands of hemodialysis patients. The availability of some of the most important medications required for hemodialysis patients, as well as the lack of insurance coverage for these drugs, were also identified as major concerns by all participants. This lack of help has become a barrier, causing carers to become more stressed and anxious, and they are asking more attention from authorities as a result (31-33). All of this is in line with the findings of the current study. In contrast to the findings of this study, earlier research in industrialized nations has found that caregivers are supported by the government in many ways, allowing them to continue caring for their patients while remaining financially secure and in good mental health $(34,35)$.

\section{Limitation}

One of the study's shortcomings was that it didn't take into account the participants' personal traits, as well as their intellectual and mental occupations during the interview procedure, which could have influenced how they answered. In this regard, the researcher attempted to pay attention to their conditions prior to conducting the interviews and to remove this constraint by offering thorough explanations to the participants and by maintaining a sincere atmosphere to the greatest extent feasible.

\section{Conclusion}

Caregivers of hemodialysis patients reported two separate and conflicting experiences when they thought they did not have enough material and spiritual assistance from their family, the community, or the authorities. Some people saw progress and excelled, believing that living in such conditions enabled them to mature and expand intellectually, whereas others saw captivity, being trapped, and sacrifice. More in-depth research is required to discover the factors that influence such diverse insights. "What is the difference between the individuals and their insights that, under similar circumstances, one believes he/she has evolved while the other believes he/she is the perfect victim and loser?" is the question that arises. More in-depth research is required to discover the factors that influence such diverse insights.

\section{References}

1. Jafari, H., Ebrahimi, A., Aghaei, A., Khatony, A. The relationship between care burden and quality of life in caregivers of hemodialysis patients. BMC Nephrol 2018;19:321.

2. Mehmood, Y., Ghafoor, S., Ashraf, M., Riaz, H., Atif, S., Saeed, M. Intradialytic complications found in patients at a tertiary care hospital. Austin J Pharmacol Ther 2016;4(1):1079.

3. Shahgholian, N., Yousefi, H. The lived experiences of patients undergoing hemodialysis with the concept 
of care: a phenomenological study. BMC Nephrol 2018;19(1):338.

4. Atik, D., Karatepe, H., Karatepe, C., Demir, S., Çınar, S., Sökmen, S. The effect of gender on the complications, pain intensity and pain management in hemodialysis patients. Int $\mathbf{J}$ Res Med Sci. 2016;4(5):1490-5.

5. Ashghali Farahani, M., Ghane, G., Sydfatemi, N., Hagani, H. Effect of Educational Program on the Burden of Family Caregivers of Hemodialysis Patients. Evidence Based Care 2016;6(1):7-18.

6. Sajadi, S.A., Ebadi, A., Moradian, S.T. Quality of Life among Family Caregivers of Patients on Hemodialysis and its Relevant Factors: A Systematic Review. Int $\mathrm{J}$ Community Based Nurs Midwifery 2017;5(3):206-18.

7. Salehitali, S., Ahmadi, F., Hasanpour Dehkordi, A., Noorian, K., Fereidooni-Moghadam, M., Zarea, K. Progressive exhaustion: A qualitative study on the experiences of Iranian family caregivers regarding patients undergoing hemodialysis. Int $\mathbf{J}$ Nurs Sci 2018;5(2):193-200.

8. Alnazly, E.K. Burden and coping strategies among Jordanian caregivers of patients undergoing hemodialysis. Hemodial Int 2016;20(1):84-93.

9. Farzi, S., Farzi, S., Moladoost, A., Ehsani, M., Shahriari, M, Moieni. M. Caring Burden and Quality of Life of Family Caregivers in Patients Undergoing Hemodialysis: A Descriptive-Analytic Study. Int J Community Based Nurs Midwifery 2019;7(2):88-96.

10. Avşar, U., Avşar, U.Z, Cansever, Z., Yucel, A., Cankaya, E., Certez, H., Keles, M., Aydinli, B., Yucelf, N. Caregiver Burden, Anxiety, Depression, and Sleep Quality Differences in Caregivers of Hemodialysis Patients Compared With Renal Transplant Patients. Transplant Proc 2015;47(5):1388-91.

11. De Pasquale, N., Cabacungan, A., Ephraim, P.L., Lewis-Boyér, L., Powe, N.R., Boulware, L.E. Family Members' Experiences With Dialysis and Kidney Transplantation. Kidney Medicine 2019,1(4):171-179.

12. Burns, N., Grove, S. The Practice of Nursing Research: Conduct, Critique, and Utilization: Elsevier/Saunders; 2005

13. Grove, S.K., Gray, J.R., Burns, N. Understanding Nursing Research E-Book: Building an EvidenceBased Practice: Elsevier Health Sciences; 2018.

14. Polit, D.F., Beck, C.T. Essentials of Nursing Research: Appraising Evidence for Nursing Practice: Wolters Kluwer; 2018.

15. Holloway, I., Galvin, K. Qualitative Research in Nursing and Healthcare: Wiley; 2016.

16. Burns, N., Grove, S.K. Understanding Nursing Research - eBook: Building an Evidence-Based Practice: Elsevier Health Sciences; 2010.
17. Streubert, H.J., Carpenter, D.R. Qualitative Research in Nursing: Advancing the Humanistic Imperative: Wolters Kluwer Health/Lippincott Williams \& Wilkins; 2011.

18. Wightman, A., Zimmerman, C.T., Neul, S., Lepere, K., Cedars, K., Opel, D. Caregiver Experience in Pediatric Dialysis. Pediatrics 2019;143(2):e20182102.

19. Hoang, V.L., Green, T., Bonner, A. Informal caregivers' experiences of caring for people receiving dialysis: A mixed-methods systematic review. J Ren Care 2018;44(2):82-95.

20. Khorsandi, F., Parizad, N., Feizi, A., Hemmati MaslakPak, M. How do parents deal with their children's chronic kidney disease? A qualitative study for identifying factors related to parent's adaptation. BMC Nephrology. 2020;21(1):509.

21. Rocha, R.C.N.P., Pereira, E.R., Silva, R.M.C.R.A., de Medeiros, A.Y.B.B.V., Refrande, S.M., Refrande, N.A. Spiritual needs experienced by the patient's family caregiver under Oncology palliative care. Rev Bras Enferm 2018;71(Supl. 6):2635-2642.

22. Barbosa, R.M.d.M., Ferreira, J.L.P., Melo, M.C.B.d., Costa, J.M. Spirituality as a coping strategy for families of adult patients in palliative care. Rev SBPH 2017;20(1):165-82.

23. Greenwood, N., Smith, R. Barriers and facilitators for male carers in accessing formal and informal support: A systematic review. Maturitas 2015;82(2):162-9.

24. Greenwood, N., Mackenzie, A., Harris, R., Fenton, W., Cloud, G. Perceptions of the role of general practice and practical support measures for carers of stroke survivors: a qualitative study. BMC Fam Pract 2011;12:57.

25. Mieto FSR, Bousso RS. The mothers' experiences in the pediatrics hemodialysis unit. Bras $\mathrm{J}$ Nefrol 2014;36(4):460-8.

26. Tong, A., Sainsbury, P., Chadban, S., Walker, R.G., Harris, D.C., Carter, S.M., Hall, B., Hawley, C., Craig, J.C. Patients' Experiences and Perspectives of Living With CKD. Am J Kidney Dis 2009;53(4):689700.

27. Ghane, G., Farahani, M., Seyedfatemi, N., Haghani, H. The effect of supportive educative program on the quality of life in family caregivers of hemodialysis patients. J Educ Health Promot $2017 ;: 80$.

28. Eirini, G., Georgia, G. Caregivers of Patients on Haemodialysis. Caregiving and Home Care 2018:7584.

29. Pourghaznein, T., Heydari, A., Manzari, Z., ValizadehZare, N. "Immersion in an Ocean of Psychological Tension:" The Voices of Mothers with Children Undergoing Hemodialysis. Iran J Nurs Midwifery Res 2018;23(4):253-60. 
30. Mthembu, T.G., Brown, Z., Cupido, A., Razack, G., Wassung, D. Family caregivers' perceptions and experiences regarding caring for older adults with chronic diseases. South African Journal of Occupational Therapy. 2016;46(1):83-88.

31. Essue, B., Kelly, P., Roberts, M., Leeder, S., Jan, S. We can't afford my chronic illness! The out-ofpocket burden associated with managing chronic obstructive pulmonary disease in western Sydney, Australia. J Health Serv Res Policy 2011;16(4):22631.

32. Jeon, Y-H., Essue, B., Jan, S., Wells, R., Whitworth, J.A. Economic hardship associated with managing chronic illness: a qualitative inquiry. BMC Health Serv Res 2009;9:182.

33. Oyegbile, Y.O., Brysiewicz, P. Exploring caregiver burden experienced by family caregivers of patients with End-Stage Renal Disease in Nigeria. Int J Africa Nurs Sci 2017;7:136-43.
34. Northouse, L.L., Katapodi, M.C., Schafenacker, A.M., Weiss, D. The Impact of Caregiving on the Psychological Well-Being of Family Caregivers and Cancer Patients. Semin Oncol Nurs 2012;28(4):23645.

35. Epiphaniou E, Hamilton D, Bridger S, Robinson V, Rob G, Beynon T, Higginson, I., Harding, R. Adjusting to the caregiving role: the importance of coping and support. Int $\mathbf{J}$ Palliat Nurs 2012;18(11):541-5. 